\title{
Focal placenta accreta
}

\author{
Fariba Minaee $^{1,}$, Manoochehr Salimian², Monireh Dehghani Arani ${ }^{3}$ \\ ${ }^{1}$ Gynecology, Theran University of Medical Sciences, Tehran, Iran \\ ${ }^{2}$ Medical, Kashan University of Medical Sciences, kashan, Iran \\ ${ }^{3}$ Biostatistics, Faculty of Paramedicine, Shahid Beheshti University of Medicine Sciences, Tehran, Iran
}

\section{Email address:}

f_minaee2005@yahoo.com (F. Minaee)

\section{To cite this article:}

Fariba Minaee, Manoochehr Salimian, Monireh Dehghani Arani. Focal Placenta Accreta. Journal of Gynecology and Obstetrics. Vol. 2, No. 3, 2014, pp. 46-48. doi: 10.11648/j.jgo.20140203.14

\begin{abstract}
Most of the time in order to control post partum hemorrhage due to abnormal placental connection, hysterectomy is necessary. In this case report, Baker Report balloon was used to save the patient. Mother morbidity and mortality can be prevented with this way. Abnormal placental connection incidence containing. Accrete, increta and percreta is $1 / 7000$. Severe placenta percreta causes high mortality and morbidity. Placenta percreta is accompanying with praevia in $75 \%$ of cases. $(1,2)$
\end{abstract}

Keywords: Placenta Accrete, Placenta praevia, Fetal Anomalies, Previous Caesarean Section, Baker Balloon

\section{Introduction}

Post partum hemorrhage is still one of the important reasons of maternal mortality in developed and developing countries. (3)

Also, it consider as an important factor of maternal morbidity in those countries.

interfere Forming inferior segment and dilation while the placenta is located at internal os of cervix causes inevitable rupture of placenta. The inherent impotency of inferior or myometer fibers contracting ruptures vessels can aggravate bleeding. (4)

Placenta praevia can accompanied with placenta Accrete or it's more advanced forms meaning placenta increta or percreta. Connecting has been reported in this way, abnormal connection and placental location at inferior segment due to infirmity of decidual development can be predicated.

5.4 of placenta praevia is accompanying with $7 \%$ of abnormal placental connection. (4)

Placenta praevia comes with Painless third trimester bleeding. The incidence is 3-5 per 1000 pregnancy which is going higher due to raising caesarean section rate.(5)

The risk of placenta Accrete is 54 times. However, it is 8.3 times if serum $\mathrm{Alf}=2.5 \mathrm{MOM}$ or it increases to 3.2 times if mother age $\geq 35$.(4)

It can be diagnosed by abdominal ultrasound or MRI during Pregnantcy. Also it controls with post partum hysterectomy, internal iliac tubal ligation, pressure sutures and cervical packing.

\section{Case Report}

A 32 years pregnant woman with 15-16 WK age and post history of Cesarean section with legal permission from forensic medicine referred to Shahid Rajaee hospital of Aran and Bidgol for abnormal fetus abortion.

1th day hospital: Cervical Ripening with 2 Misoprostol vaginal tablets was used which was unsuccessful.

2th day hospital: Induction applied using $100 \mathrm{IU}$ oxytocin.

3th day hospital: 200 IU oxytocin and 2 Misoprostal vaginal tablets were prescribed.

Patient referred to OR due to Severe vaginal bleeding and pre shock position for Dilation and curettage. 6 units packed cell and 5 Misoprostol tablet were used during Dilation and curettage which controlled abnormal bleeding.

5th day: ultrasound reported pregnancy residue and Dilation and curettage was performed.

Intra uterus balloon, 4 units packed cell and 2 Misoprostol vaginal tablets were used for bleeding control. 6th day: patient was released from hospital with $\mathrm{Hb}=$ 10.8

Placenta praevia was reported in primary ultrasound was done by physician while it had not reported in following ultrasounds with fetus abnormal like Gastrochesia.

Patient Severe bleeding during dilation was because of placenta praevia. She was hospitalized in 7 WKS 
pregnancy for spotting.

She come back to the hospital with vaginal bleeding and fever a week after Dilation and curettage.

She treated using Clindamicin and Gentamicin $\mathrm{Hb}=11.3$, $\mathrm{BHCG}=3360$ and a $55-56 \mathrm{~mm}$ mass was reported in ultrasound at inferior segment suspicious to hematoma or Molar pregnancy.

However, Mollar pregnancy was rejected.

Two weeks after Dilation and curettage, ultrasound reported a $67 \mathrm{~mm}$ mass inferior segment which talked about pregnancy residual or focal rupture of myometer $(\mathrm{Hb}=10.6, \mathrm{BHCG}=2550)$

CT reported a normal uterus with no pathological sign. Pathology test from Dilation and curettage sample reported normal pregnancy residual.

Three weeks after Dilation and curettage, ultrasound reported a $1075 \mathrm{~mm}$ hematoma at inferior segment, inside the cavity with myometer penetration focal myometer rupture was discussed in previsions ultrasound $(\mathrm{Hb}=5.13$, $\mathrm{BHCG}=6120$ )

4 weeks after $\mathrm{s}$, MRI reported a hetrogen mass $109 \times 75 \times 88$ size at inferior segment which damaged cervix.

To control intra hematoma bleeding located at cervix laparotomy with high risk hysterectomy was planned. Patient insisted to save her uterus.

A Gynecologist with a surgeon made laparatomia but cervix rupture didn't noticed, It was fully dilated expressing intra cervical mass. A piece of placenta $(3 \times 4 \mathrm{~cm})$ was attached on cervix with myometer infiltration (inferior segment, previous Cesarean section incision).

8 units packed cell and 13 misoprostal tablets were Prescribed.

Baker Balloon was inserted to control bleeding from placental location. finally, bleeding stopped, Balloon was inserted from vagina and enlarged using $100 \mathrm{cc}$ normal saline. Leading packing bleeding site, then incision was repaired and patient referred to ICU.

24 hours later, some ballon liquid was extracted, but because of bleeding, it enlarged again until bleeding stopped.

Despite of patient alertness, she was hospitalized for 20 days until bleeding completely stopped. Balloon exited even though uterus tissue necrosis was discussed, we could save patient uterus using Baker ballon despite of focal accrete in inferior Segment (previous Cesarean section incision location) and severe bleeding. Finally, patient was released from hospital 40 days after Dilation and curettage in good situation.

\section{Discussion}

Placenta percreta is abnormal placental replacement with severe adhesion to the uterus (6); its pathologic sign is lack of decidual Basalis or incompetency of Nitabuch layer. (4)

Placenta percreta risk factors are: placenta accrete on previous Cesarean section and scar, multipartite, pregnancy age, smoking, alcohol and addiction history. $(6,7)$
In this case, previous Cesarean section and placenta praevia were risk factors.

Placenta praevia is there times more in women with previous Cesarean section. Its rate increases with the number of Cesarean section.

$1.9 \%$ after 2 times Cesarean section and $4.1 \%$ after 3 times Cesarean section or more(4).Placenta praevia is leading to maternal or fetal serious complications $(5 / 1000$ Pregnancy) maternal mortality rate is $4.7 \%$

It is indicated that smoking has greater rate (26\%).Previous abortion (16\%) and previous Cesarean section $(5-10 \%)$

The main reason is decreasing placenta and uterus oxygenation. Low blood flow and increasing surface might be needed for placenta connection to the inferior segment. (8).

Placenta accrete describes any placenta replacement which comes with abnormal strong adhesion to the uterus because of complete or Proportional lack of decidual Basalis and incomplete developing of fibrotoeid (Nitabuch) layer.

In accrete placental piles attach to the myometer in increta, they aggregate to the myometer in percreta, they pass through myometer.

Abnormal adhesion could occur in whole cotilidons (complete accrete), few or some cotilidons (Proportional accrete) or only one cotilidons (Focal Placenta accrete)(4)

In this case, we faced to a focal accrete with previous Cesarean section history.

The accrete and increta rate Increases with the rasing Cesarean section rates. (4)

Abnormal adhesion occurs when disioual Basalis forming was incomplete.

Following situation contains of placental replacement at inferior segment in previous Cesarean section incision or previous Curettage women (1/2) 0 Gravid $\geq 6$ (1/4). (4)

The risk of accrete increases 54 times (praevia) 8.3 times (EP),3.9 times (Free BHCG),3.2 times( $\geq 35$ years).(4)

In this case focal accrete came with anomal fetus. It is possible that increta detect prior to delivery.

The ultrasound sensivity to detect accreta is only $33 \%$ while it is $100 \%$ in multicolor Doppler ultrasound. Its positive prediction is $78 \%$.Using 3D ultrasound or Doppler in perccerta detecting has been recommended. MRI has only $38 \%$ sensitivity in accrete detection. Raising D dimmer may affect on accrete prediction. Possibly, this finding is a thorfoblast aggregation reflexion into myometer or neibouring tissue.(4)

For treatment in Severe bleeding, immediate blood replacement and hysterectomy is necessary.

Other bleeding methods are:

Internal iliac artery ligation or uterus artery mobilization, using of agglutination or arong ray. (4)

The most common conservative treatment method was feasible placental manual exiting, then uterus packing in the past.

If bleeding is not impressive, placenta leaves at it's place 
and weekly methotrexate prescribed until it will automatically reject from vagina one month later.

It is possible that involved cotilidon and myometer together will Severe bleeding in focal accrete.

Also, it is possible that cotilidon detach from placenta and attach to the nesting place. It accompanying with raising bleeding (immediate or delayed), this is one of the forming of placental polyp mechanism. (4)

In this case, Baker Balloon was used in focal accrete in order to control bleeding fortunately; it was a successful method was leading to save patient fertilization.

\section{References}

[1] Kent A. Management of placenta accrete.Rev.Obstet Gynecol 2009; 2(2): 127-81.

[2] O'Brien JM,Barton J,Donaldson ES.The management of placenta percreta: conservative and operative strategies. Am J Obstet Gynecol 1996;175:1632 -8.
[3] Moriya M,Kusaka H,Shimizu K,Toyoda N.Spontaneus rupure of uterus caused by placenta percreta at 28 weeks of gestation :a case report. J Obstet Gynaecol res 1998; 24: 211-4.

[4] Lieberman JR,Fraser D,Kasis A,etal.Reduced frequency of hypertsive disorders in placenta praevia.obstet Gynecol 1991;77;83-6.

[5] Morgan K, Arulkumaran S.Antepartum haemorrhage.Currr Obstet Gynecol 2003; 13; 81-87.

[6] Zahn CM, Yeomans ER. Postpartum haemorrhage; placenta accrete,Uterine inversion and puerperal hematomas.Clin obstet Gynecol 1990;33:422-31.

[7] Faiz AS, Ananth CV.Etiology and risk factors for placenta praevia:an overview and meta-analysis of observational studies The J Matern - fet Neonat med 2003;13:175-190.

[8] Williams MA,Mittendorf R.Increasing maternal age as a determinant of placenta praevia:More important than increasing parity.J Reprod Med 1993;38:425 -8. 